\title{
Perceptual-Motor Contributions to Static and Dynamic Balance Control in Children
}

\author{
V. Hatzitaki \\ V. Zisi \\ I. Kollias \\ E. Kioumourtzoglou \\ Department of Physical Education and Sports Sciences \\ Aristotelio University of Thessaloniki \\ Greece
}

\begin{abstract}
The authors addressed balance control in children from the perspective of skill development and examined the relationship between specific perceptual and motor skills and static and dynamic balance performance. Fifty 11- to 13-year-old children performed a series of 1-legged balance tasks while standing on a force platform. Postural control was reflected in the maximum displacement of the center of mass in anterior-posterior and mediolateral directions. Simple visual, discrimination, and choice reaction times; sustained attention; visuomotor coordination; kinesthesis; and depth perception were also assessed in a series of perceptual and motor tests. The correlation analysis revealed that balancing under static conditions was strongly associated with the ability to perceive and process visual information, which is important for feedback-based control of balance. On the other hand, when greater task demands were imposed on the system under dynamic balancing conditions, the ability to respond to the destabilizing hip abductions-adductions in order to maintain equilibrium was associated with motor response speed, suggesting the use of a descending, feedforward control strategy. Therefore, like adults, 11- to 13-year-old children have the ability to select varying balance strategies (feedback, feedforward, or both), depending on the constraints of a particular task.
\end{abstract}

Key words: balance, feedback vs. feedforward control, perceptualmotor skills

$\mathbf{P}$

ostural control has been closely associated with the ability to correctly perceive the environment through peripheral sensory systems, as well as to centrally process and integrate proprioceptive, visual, and vestibular inputs at the level of the central nervous system (CNS). That ability enables the CNS to form appropriate muscle synergies needed so that equilibrium can be maintained (Teasdale, Lajoie, Bard, Fleury, \& Courtemanche, 1992). Although there is considerable evidence concerning the importance of sensory organization abilities for balance control in children (Assaiante \& Amblard, 1992; Sundermier \& Woollacott, 1998; Sundermier, Woollacott, Roncesvalles, \& Jensen,
2000; Sveistrup \& Woollacott, 1996), the exact nature of those mechanisms in terms of skill development is not yet well explored. The focus in most studies has been on looking at postural responses to visual or proprioceptive perturbations (Assaiante, \& Amblard, 1992; Sundermier et al., 2000) or to sensory conflict situations created by the combination of the two, such as the moving room paradigm (Sveistrup \& Woollacott, 1996). On the other hand, both researchers and clinicians rely on qualitative scales of movement performance such as the Bruininks-Oseretsky test for motor proficiency or the Motor Skills Inventory (Werber \& Bruininks, 1988) to assess motor skill development and balance in children. Although those scales are useful for establishing an overall level of performance as compared with norms, they do not provide any insight into how one might use specific perceptual and motor skills to predict behavior on static or dynamic balance tasks. In the present study, we attempted to address balance control in children from the perspective of skill development and to examine how specific perceptual and motor skills are related to static and dynamic balance performance.

Before considering perceptual and motor skill contributions to balance control, one must properly address the issue of the different strategies used by the CNS in order to maintain equilibrium, depending on the nature of the task, static or dynamic. For example, quiet standing is said to be controlled by sensory feedback on the basis of a closed-loop system (Nashner, 1976) in which the center of foot pressure moves in phase with the center of mass (Winter, Patla,

Correspondence address: Vassilia Hatzitaki, Department of Physical Education and Sports Sciences, Aristotelio University of Thessaloniki, Thessaloniki, 540 06, Greece. E-mail address: vaso1@phed.auth.gr 
Prince, Ishac, \& Gielo-Perczak, 1998), and integration of visual and proprioceptive inputs is needed for that control (Massion \& Woollacott, 1996). The importance of visual cues in maintenance of static posture has been well demonstrated, particularly in children, who use them to visually monitor their body during posture (Gallahue \& Ozmun, 1998; Riach \& Hayes, 1987). In addition, proprioceptive information from virtually all areas of the body is coprocessed and integrated at a central level so that it can contribute to a stable posture (Jeka, Oie, \& Kiemel, 2000; Kavounoudias, Gilhodes, Roll, \& Roll, 1999; Roders, Wardman, Lord, \& Fitzpatrick, 2001; Slijper \& Latash, 2000).

On the other hand, balancing under dynamic task conditions requires the use of feedforward control (Horak \& Nashner, 1986; Lacquaniti \& Maioli, 1989). With feedforward control, postural disturbances are predicted, and those predictions result in anticipatory postural adjustments (APAs) that enable the mover to maintain stability (Massion, 1992). In that case, equilibrium control is of a more reflexive nature and depends on the ability to rapidly transform perturbations of proprioceptive or vestibular origin into proper motor responses, an ability that has been linked to an adequately functioning reaction time process (Lord, Clark, \& Webster, 1991; Woollacott, Shumway-Cook, \& Nashner, 1986). The importance of cognitive function for the organization and integration of available sensory information under both static and dynamic balancing conditions has also been well acknowledged (Schmidt, 1982; Shumway-Cook \& Woollacott, 2000; Vuilerme, Nougier, \& Teasdale, 2000; Woollacott, Moore, \& Hu, 1992). Nevertheless, the differential contributions of skills such as depth perception and reaction time or cognitive skills such as attention to the performance of static or dynamic balance tasks have not been largely explored. Because task constraints are different for static and dynamic balance, we hypothesized that, depending on whether task constraints were static or dynamic, different perceptual and motor skills would be associated with balance, reflecting feedback or feedforward mechanisms of balance regulation, respectively.

The selection of the appropriate balance strategy in each case not only depends on task constraints and environmental demands but is also a function of neural maturation and experience. A feedback-based system in the control of balance appears very early in life, as confirmed by experimental evidence showing that postural response synergies triggered by sensory perturbations are present as early as 15-31 months and have latencies comparable with those of adults (Shumway-Cook \& Woollacott, 1985). On the other hand, a child's ability to apply feedforward control and initiate an APA to upcoming perturbations greatly depends on the ability to control gravity and inertial forces (Berger, Quintern, \& Dietz, 1987; Grasso, Assaiante, Prevost, \& Berthoz, 1998; Schmitz, Martin, \& Assaiante, 1999) and to move the head independently of the trunk (Assaiante \& Amblard, 1995), skills that develop later, between 6 and 10 years of age. However, the presence of a developmental anticipatory behavior is task specific and is shaped through "exercise and training in the specific environment" (Haas \& Diener, 1988 , p. 58), and depends on whether the perturbation is externally imposed or self-initiated (Riach \& Hayes, 1987).

We designed the present study to examine whether 11- to 13-year-old children are capable of selecting the appropriate balance strategy depending on task constraints, static or dynamic. The child's ability to select a feedforward- or feedback-based control mechanism would be reflected in the differential contribution of specific perceptual-motor skills to balance performance depending on whether the particular task conditions are static or dynamic. Children performed a series of static and dynamic balance exercises during one-legged stance on a force platform, which allowed us to quantify balance characteristics. They were then assessed in a series of perceptual-motor tests involving reaction time, depth perception, sustained attention, kinesthesis, and visuomotor coordination measures. We used a multiple correlation approach to investigate balance on the basis of a multilayered or multifactorial spectrum of selected motor, perceptual, and cognitive skills.

\section{Method}

\section{Participants}

Fifty boys ( $11 \pm 1.7$ years of age) volunteered to participate in the present study following informed consent. None of the children had a pathological disorder associated with either the visual or vestibular system or had been involved in sports activities in which a single-limb stance was used. At the beginning of the experimental session, participants were asked to declare their dominant kicking leg. All balance exercises requiring one-legged stance were performed on the limb contralateral to the dominant kicking leg.

\section{Experimental Task and Protocol}

Static and dynamic balance tests. We achieved static balance conditions by asking participants to stand on the force platform on one leg (single-limb stance). The nonsupporting limb was flexed at the knee, with the foot's plantar surface stabilized on the knee of the supporting leg ("stork" balance; see Figure 1a). At the go signal, each child was required to elevate his heel and to keep his balance for a minimum duration of $5 \mathrm{~s}$, narrowing his base of support to an area of a few centimeters.

In the dynamic balance test, the children had to stand on the force platform on one leg while performing two different exercises with the nonsupporting limb (Figure $1 \mathrm{~b}$ and $\mathrm{c}$ ). The first exercise involved repetitive hip flexion and extension of the swinging limb, and the second required the children to perform hip abduction-adduction in a similar way. Hip flexion-extension induced a perturbation in the anterior-posterior $(x)$ direction, and hip abduction-adduction similarly introduced a perturbation in the mediolateral $(y)$ direction. Participants were instructed to perform both exer- 
a) STATIC balance

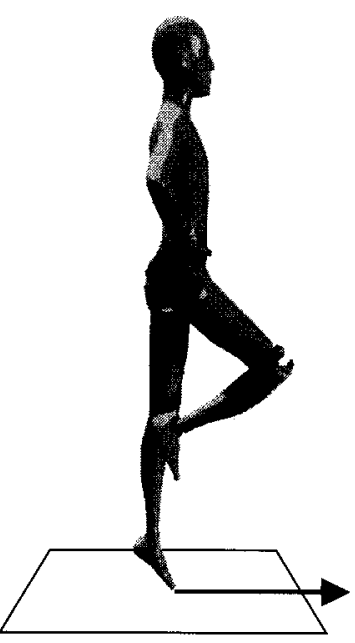

b) DYNAMIC balance I

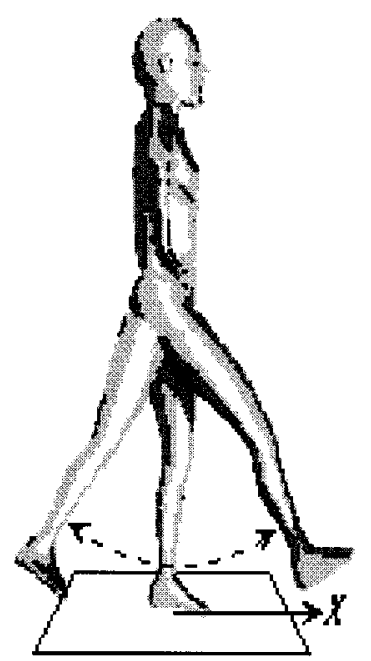

c) DYNAMIC balance II

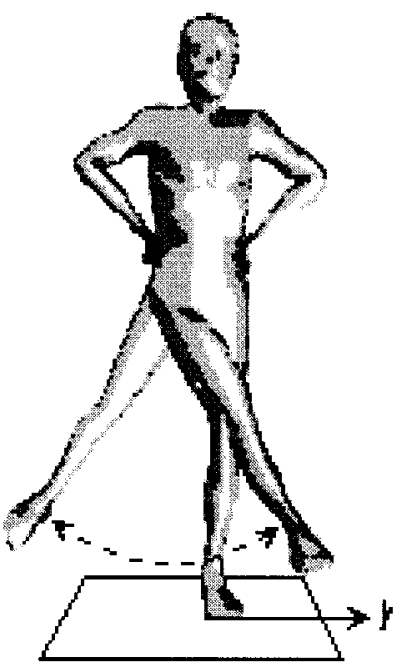

FIGURE 1. Schematic representation of the static and dynamic balance tasks: a. Static balance. b. Dynamic balance I: flexion-extension. c. Dynamic balance II: abduction-adduction.

cises as fast as possible and at their full range of motion. Dynamic balance exercises were performed repeatedly over a time period of $5 \mathrm{~s}$. Each participant performed two test trials following a practice trial, and, on the basis of maximum force output, we selected the better of the two trials for further analysis. Those trials in which the child was unable to sustain his balance for a period of $5 \mathrm{~s}$ were excluded from further analysis. Five of the children tested were unable to keep their balance for $5 \mathrm{~s}$ in one of the static or dynamic balance exercises, and as a result of that inability, their data were not considered in the subsequent analysis.

Perceptual-motor tests. In addition to balance testing, the children performed the following series of tests:

1. They performed a simple reaction time test to a visual stimulus (RT visual) by using the Reaction Test software of the VIENNA Test Instrument System (Schuhfried, 1996). Participants were seated comfortably behind a monitor, with the index finger of their dominant hand resting on a touch-sensitive key. As soon as a black circle turned yellow, they had to press a bar button located $8 \mathrm{~cm}$ above the resting key. The release of the resting key, with consequent pressing of the reaction button at stimulus appearance, was considered a correct reaction. Reaction time was defined as the interval between the onset of the stimuli and the release of the resting key. After oral instructions and four subsequent correct responses in the practice phase, the children performed 24 trials. Intertrial interval was varied between 2.5 to $6.5 \mathrm{~s}$. The mean reaction time of the correct reactions was recorded.

2. The children performed a simple reaction test to an auditory stimulus (RT auditory) with a different software version of the VIENNA Test Instrument System. The procedure and recording were the same as before. However, the auditory stimulus in that test was a tone (frequency of 2000 $\mathrm{Hz}$ ) presented on earphones via the system's interface.

3. The children performed a discrimination reaction time test (DRT) with another version of the Reaction Test software. A red light, a combined red-yellow light, a tone, and combinations of the three were presented alternatively on the monitor. The children had to react only to the critical stimulus, which was the simultaneous onset of red and yellow lights. The response procedure and recording were exactly the same as with the simple reaction time test.

4. The children performed a choice reaction time test (CRT) with the Determination Test software of the VIENNA Test Instrument System. The children were seated behind a monitor and had to react to seven different visual and two different auditory stimuli by using their upper and lower limbs. They had to press one of five round colored buttons, arranged in a semicircle on the working panel, as soon as a circle of the corresponding color appeared on the screen. Finally, they were also required to press a left or right foot pedal as soon as a white rectangle appeared on the corresponding side of the screen. The frequency of stimulus presentation was controlled by the child's working speed. After oral instruction and separate instruction phases for the three types of stimuli (round circle, white rectangles, and tones) and the combination of the three, the children performed the test for $4 \mathrm{~min}$. The mean reaction time (in milliseconds) and the total number of correct and incorrect responses were recorded.

5. The children performed a sustained attention test 
(ATN), using the continuous attention test software of the VIENNA Test Instrument System. The children were seated comfortably behind a monitor, with the index finger of the dominant hand resting on the lower bar button of a working panel. A row of seven triangles, pointing upward or downward, was presented on the screen with irregular jumps. The children had to press the bar button as soon as they realized that three of the triangles were pointing downward (critical stimulus). After successive completion of a practice phase, the children performed the test for $10 \mathrm{~min}$. During that period, 80 critical stimuli out of 400 , with a length of $1.5 \mathrm{~s}$ each, were presented. The numbers of correct and incorrect responses as well as the mean reaction time (in milliseconds) for those responses were recorded.

6. The children performed a line-tracking test (LT) with the Motor Performance Series of the VIENNA Test System. Using a stylus, the children had to track a path on a vertical work board without touching the sides or the rear. After one practice trial, they performed one trial, and the total time of trial completion and numbers of errors were recorded.

7. The children performed a depth perception test (DPF, DPB) with the Electric Depth Perception Tester (Takei Scientific Instruments, Tokyo, Japan). The children were seated, and their chin was stabilized so that they could see a particular visual field through a slot located $120 \mathrm{~cm}$ away at eye level. Through that slot, they could see a dowel moving forward or backward at a speed of $50 \mathrm{~mm} / \mathrm{s}$, and they had to press a button as soon as they realized that the dowel was aligned with two other dowels located bilaterally $20 \mathrm{~cm}$ away from the moving dowel. Although the test instrument is designed so that depth perception can be measured, it is set up so that performance outcome also involves a significant motor reaction time as well as an anticipatory component. The influence of motor response speed on performance was eliminated, however, because the movement of the experimental dowel was very slow $(50 \mathrm{~mm} / \mathrm{s})$; in addition, the dowel's motion was always available to the children's sight, suggesting involvement of an anticipatory mechanism as part of the response. To further eliminate the possibility that a delay in pressing the button would be attributed to an incorrect motor response, we gave the children one practice trial to make sure that they were acquainted with the motor task and could control their movement so that they could get the result they wanted. A second practice trial was given in instances of bad movement control. After practice, participants proceeded to perform four trials in each moving direction of the dowel (forward and backward). Distance error from the alignment point was recorded for each trial in millimeters, and a mean of those values was calculated separately for the forward and backward movements.

8. The children performed a kinesthetic sense test with the kinesthesiometer (Lafayette Instrument Co., Lafayette, IN). The children were seated in parallel with a horizontal lever that rotated about the vertical axis. The children placed their dominant forearm on the lever so that the axis of rotation was aligned with the elbow joint. At the starting position, the forearm was flexed $90^{\circ}$ at the elbow. Offering manual help, the instructor guided the children' arm so that it moved the lever $60^{\circ}$ into extension, which signified a position that corresponded to a $150^{\circ}$ elbow angle. That movement was repeated for a second time, with the children blindfolded. They were then instructed to move the lever to the desired target position. which corresponded to a $150^{\circ}$ elbow angle. After two practice trials, one with eyes open and the other blindfolded, they performed eight test trials blindfolded. Lever position was recorded for each trial, and the mean absolute deviation from the $150^{\circ}$ angle was calculated (mean error).

\section{Analysis of Balance}

An AMTI force platform (Model OR6-5-1000; Advanced Mechanical Technology, Inc., Watertown, MA) recorded at a rate of 50 samples per second the ground reaction forces $(F x, F y$, and $F z$ ) exerted on the support foot during performance of the balance exercises. A representative trial, which depicts the ground reaction force components during performance of the flexion-extension exercise, is shown in Figure 2. We used a simplified method based on force platform measurements in order to estimate the maximum displacement of the center of mass (COM) in the anterior-posterior $(x)$, mediolateral $(y)$, and vertical $(z)$ directions during performance of the balance exercises (Shimba, 1984; Zatsiorksy \& King, 1998). In that method, one uses the three ground reaction force components $(F x, F y$, and $F z)$ in order to estimate COM position. Because the ground reaction force component in each direction is proportional to the acceleration of the COM in the respective direction, its second integral represents the COM displacement in each direction. One estimates the integration constants by using a technique, called zero-point-to-zero-point integration, which is based on the postulate that center of foot pressure (COP) and COM positions coincide when $F x$ is zero. The double integration of $x^{\prime \prime}(t)=F x(t) / m$ starts at the time when $F x$ is zero and continues until $F x$ is again zero. The same procedure continues from one zero point to the next zero point. That technique was analytically described by Zatsiorsky and King (1998) and was found to be in good agreement with estimations of the COM position from kinematic data for situations in which dynamics are not significant (e.g., quiet stance, postural disturbances), confirming the validity of the suggested algorithm (Caron, Faure, \& Brenière, 1997; Zatsiorsky \& King, 1998). We subtracted the positive value of the maximum COM displacement estimated by using the aforementioned technique from the subsequent maximum negative COM position in order to estimate the maximum amplitude of the COM oscillation in the anterior-posterior $(\mathrm{A} / \mathrm{P})$, mediolateral $(\mathrm{M} / \mathrm{L})$, and vertical directions. That value was used as an index of postural stability during performance of static and dynamic balance tasks. 


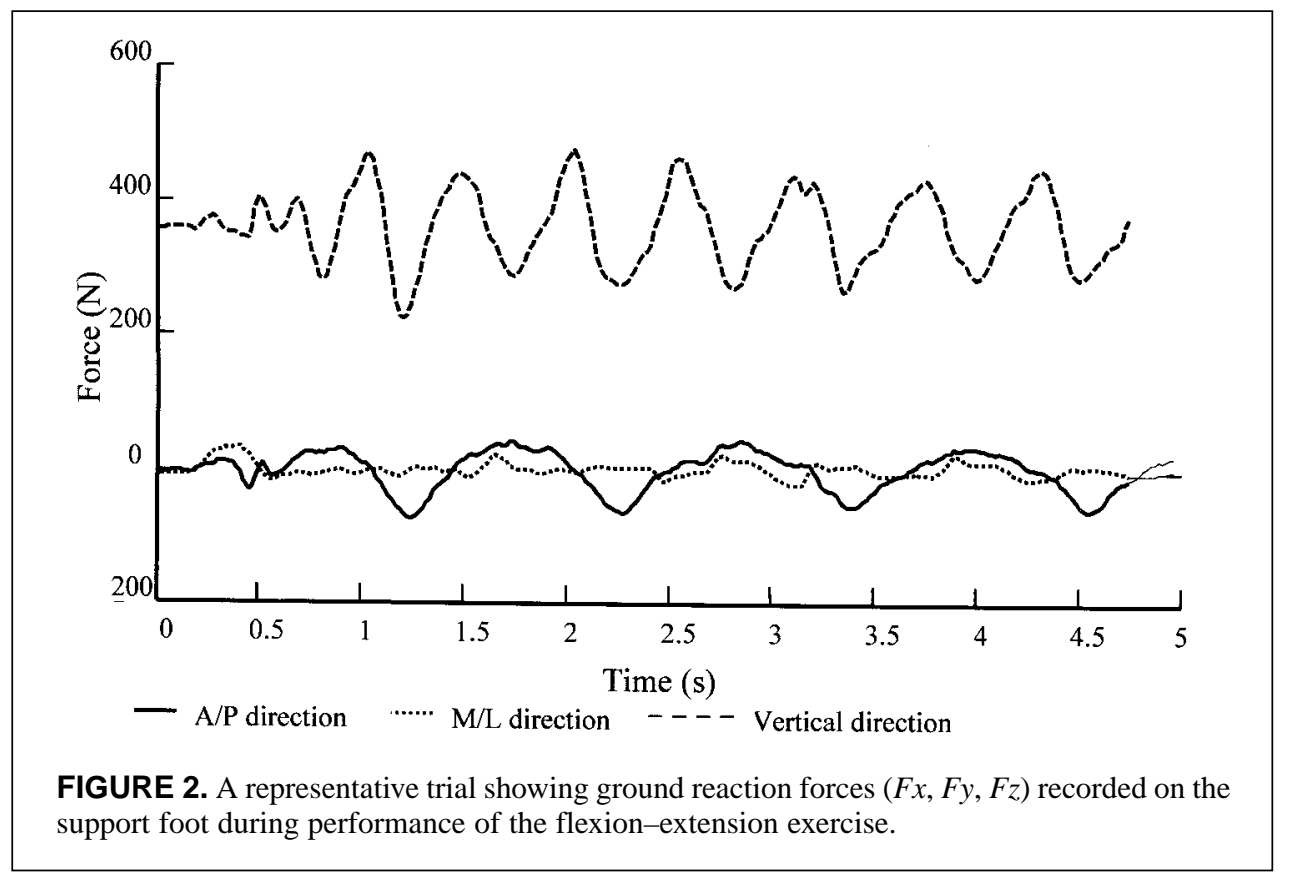

\section{Statistical Analysis}

We investigated the relationship between balance and perceptual-motor abilities by using multiple correlation and linear regression analysis techniques. All reaction time, depth perception, attention, kinesthesis, and visuomotor coordination measures examined in the present study were initially placed in a simple correlation (Pearson product moment), with the postural stability index defined as the maximum amplitude of the COM oscillation in each of the three directions. Following correlation, we formulated a multiple linear regression model to identify those perceptual-motor variables that could predict static and dynamic balance. We included in the equations only those variables found to be significantly correlated with balance, and we used stepwise regression to test the model's power in predicting static and dynamic balance from the perceptualmotor abilities examined in the present investigation.

\section{Static Balance}

\section{Results}

During performance of the static balance test (the stork balance), the maximum shift of the COM had mean values of $5.2 \mathrm{~cm}, 4.6 \mathrm{~cm}$, and $3.5 \mathrm{~cm}$ in the A/P, M/L, and vertical directions, respectively (Table 1). The analysis revealed that postural stability, expressed by the maximum amplitude of the COM oscillation in the M/L plane, was highly associated with depth perception $(r=.475, p<.01)$ when the visual stimuli were moving away from the children (backward direction; see Table 2). A positive correlation indicated that the greater the COM displacement, that is, the greater the instability in the $\mathrm{M} / \mathrm{L}$ plane, the greater the amount of spatial error in the depth perception test. Balance in the M/L direction, as expressed by the same index, was also signifi-

\begin{tabular}{|c|c|c|c|c|c|}
\hline \multicolumn{6}{|c|}{$\begin{array}{c}\text { TABLE 1 } \\
\text { Mean (M) and Standard (SD) Deviation } \\
\text { of the Maximum Amplitude of the Center } \\
\text { of Mass Displacement in Each Direction } \\
\text { During Performance of the Static } \\
\text { and Dynamic Balance Exercises }\end{array}$} \\
\hline \multicolumn{2}{|c|}{ Static } & \multicolumn{2}{|c|}{$\begin{array}{c}\text { Flexion- } \\
\text { Extension }\end{array}$} & \multicolumn{2}{|c|}{$\begin{array}{l}\text { Abduction- } \\
\text { Adduction }\end{array}$} \\
\hline$M$ & $S D$ & $M$ & $S D$ & $M$ & $S D$ \\
\hline \multicolumn{6}{|c|}{$A / P$ direction $(x)$} \\
\hline .0529 & .0319 & .0428 & .0218 & .0396 & .0100 \\
\hline \multicolumn{6}{|c|}{$M / L$ direction $(y)$} \\
\hline .0460 & .0246 & .0507 & .0153 & .0751 & .0268 \\
\hline \multicolumn{6}{|c|}{ Vertical direction $(z)$} \\
\hline .0358 & .0256 & .0547 & .0228 & .0584 & .0365 \\
\hline
\end{tabular}

cantly correlated with the number of errors in the LT test $(r=.482, p<.01)$, indicating that children with greater amplitude shift of the COM in the $\mathrm{M} / \mathrm{L}$ direction were prone to a greater number of errors in the visuomotor coordination test. Finally, the amplitude of the COM oscillation in the $\mathrm{A} / \mathrm{P}$ direction was moderately, yet significantly, correlated with the total number of incorrect responses $(r=.299, p \approx$ .05) in the CRT.

Using variables (LT and DPB) that showed a significant 
TABLE 2

Correlation Coefficients (Pearson Product Moment), Showing the Relationship

Between Balance (Static and Dynamic) and Reaction Time, Depth Perception, Attention, Kinesthesis, and Visuomotor Coordination Measures

\begin{tabular}{|c|c|c|c|c|c|c|c|c|c|}
\hline \multirow[b]{2}{*}{ Measure } & \multicolumn{3}{|c|}{ Static balance } & \multicolumn{3}{|c|}{ Dynamic balance I } & \multicolumn{3}{|c|}{ Dynamic balance II } \\
\hline & $x$ & $y$ & $z$ & $x$ & $y$ & $z$ & $x$ & $y$ & $z$ \\
\hline Simple RT visual & -.042 & -.006 & -.066 & .147 & -.146 & -.083 & $.409 * *$ & -.046 & -.197 \\
\hline Simple RT sound & -.020 & .015 & -.107 & .134 & -.218 & -.084 & .182 & -.019 & -.152 \\
\hline Discrimination RT & .069 & .071 & -.084 & .252 & -.123 & -.102 & $.419 * *$ & .009 & -.178 \\
\hline Choice RT (CRT) & -.090 & .076 & -.096 & .280 & -.216 & -.155 & .228 & -.118 & -.237 \\
\hline Correct responses in CRT & -.028 & -.267 & .092 & -.277 & .049 & .179 & -.245 & -.111 & -.044 \\
\hline Incorrect responses in CRT & $.299 *$ & .133 & .022 & -.088 & -.062 & .108 & -.236 & .196 & $.426 * *$ \\
\hline Attention RT (ART) & -.117 & -.082 & .061 & -.033 & -.172 & .180 & .193 & -.103 & .016 \\
\hline Correct responses in ART & .078 & -.080 & .074 & -.168 & .018 & .034 & -.178 & .192 & .102 \\
\hline Incorrect responses in ART & -.051 & .081 & -.151 & .253 & .129 & -.215 & .123 & -.052 & -.242 \\
\hline Line-tracking (no. errors) & -.030 & $.482 * *$ & -.045 & -.018 & -.114 & -.095 & .173 & -.138 & -.043 \\
\hline Depth perception forward & .256 & .160 & .283 & -.033 & .099 & .132 & .194 & .263 & .111 \\
\hline Depth perception backward & .005 & $.475^{* *}$ & -.028 & .023 & -.109 & .079 & .296 & .168 & .252 \\
\hline Kinesthesis & -.246 & -.156 & -.118 & -.084 & -.233 & -.248 & -.061 & .238 & -.074 \\
\hline$S D$ of kinesthesis & -.126 & -.205 & -.206 & .063 & -.005 & -.271 & -.127 & .048 & -.133 \\
\hline
\end{tabular}

Note. RT = reaction time, $x=$ anterior-posterior direction, $y=$ mediolateral direction, $z=$ vertical direction

$* p<.05$ (two-tailed). $* * p<.01$ (two-tailed).

relationship to the amplitude of the COM oscillation in the $\mathrm{M} / \mathrm{L}$ direction, we formulated a linear regression model to enable us to predict performance in the static balance test from the depth perception and visuomotor coordination measures (Table 3). The stepwise regression analysis revealed that a model that included both variables as predictors could explain $31.7 \%$ of the variance in the COM displacement in the $\mathrm{M} / \mathrm{L}$ direction, and that prediction was highly significant, $F(2,42)=9.29, p<.001$. The variation was accounted for by the number of errors in the line tracking, $t=2.35, p<.05$, and depth perception, $t=2.213, p<$ .05 , tests.

\section{Dynamic Balance}

In Table 1 are the total averaged maximum excursion of the $\mathrm{COM}$ in the $\mathrm{A} / \mathrm{P}, \mathrm{M} / \mathrm{L}$, and vertical directions during performance of the dynamic balance exercises. One can see that the amplitude of the COM oscillations did not exceed a maximum value of a few centimeters, even when a selfinduced perturbation caused by a forceful hip flexion-extension or abduction-adduction created a disequilibrium condition.

Most interesting, none of the perceptual-motor variables examined in the present study were significantly associated with the index of postural stability during performance of the flexion-extension exercise, which was quantified by the maximum amplitude of the COM oscillations in the A/P, $\mathrm{M} / \mathrm{L}$, and vertical directions (Table 2). By contrast, significant correlations between dynamic balance and the perceptual-motor indices were revealed when postural stability was assessed during performance of the second dynamic
TABLE 3

Stepwise Regression of Static Balance

(Amplitude of Center of Mass Oscillation

in the $y$ Direction) on Depth Perception (DP) Errors in Line Tracking

Model: $\mathrm{COM}=$ int $+\beta 1 \mathrm{LT}+\beta 2 \mathrm{DPB}, 42 d f$,

$M S=.000429, F=9.29, p<.000 * *, R^{2}=.317$

Hypothesis testing about the model parameters

$\begin{array}{lcclcl}\text { Parameters } & \beta & t & p< & \text { Partial } R & \\ \text { LT (no. errors) } & .342 & 2.350 & .024 * & .483 & \text { in } \\ \text { DPB } & .322 & 2.213 & .033 & .475 & \text { in }\end{array}$

Note. $\mathrm{COM}=$ maximum displacement of the center of mass in the mediolateral $(\mathrm{M} / \mathrm{L})$ direction $(y)$. $\mathrm{LT}=$ number of errors in the linetracking test. DPB = depth perception in the backward direction, $d f=$ degrees of freedom, $M S=$ mean square, and in indicates in the regression equation model.

${ }^{*} p<.05 . * * p<.01$.

exercise involving a perturbation in the $\mathrm{M} / \mathrm{L}$ direction induced by the forceful hip abduction-adduction. More specifically, correlation analysis revealed that maximum displacement of the COM in the $\mathrm{A} / \mathrm{P}$ direction during performance of the abduction-adduction exercise was significantly correlated with simple visual $(r=.409, p<.01)$ and discrimination $(r=.419, p<.01)$ reaction times (Table 2). A positive correlation indicated that children who displayed shorter reaction times under both simple and discrimination response conditions could better control the COM excursion during performance of the abduction-adduction exer- 


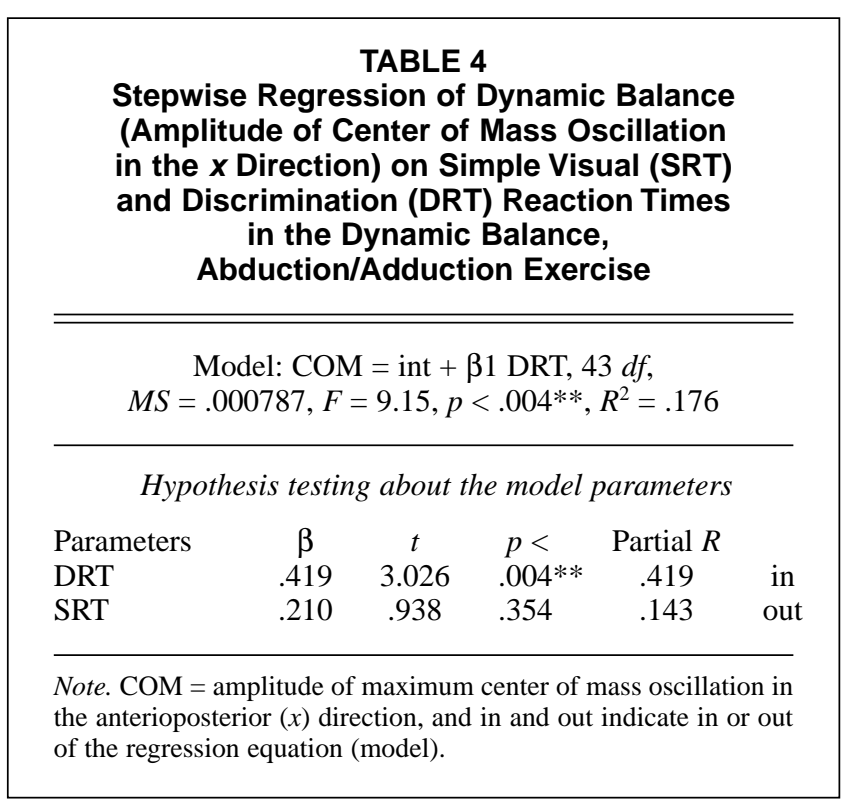

cise, which in turn resulted in a smaller amplitude of COM oscillation. In addition, vertical $(z)$ displacement of the COM during the performance of an abduction-adduction exercise was significantly associated with the number of incorrect responses in the CRT test $(r=.426, p<.01)$.

To further investigate the dependence of postural stability during dynamic balance exercise on reaction time processes, we formulated a stepwise regression model by using as predictor variables those reaction time measures showing a significant relationship to the amplitude of the $\mathrm{COM}$ oscillation in A/P direction (Table 4). The analysis revealed that a model that included discrimination reaction time (DRT), $t=3.026, p<.01$, as a predictor variable could explain $17.6 \%$ of the variation in the amplitude of the $\mathrm{A} / \mathrm{P}$ $\mathrm{COM}$ oscillation and that that prediction was highly significant, $F(1,43)=9.15, p<.01$. On the other hand, the simple reaction time $(\mathrm{SRT}), t=.938, p>.05$, did not enter the regression equation, mainly because of its significant relationship to DRT $(r=.784, p<.01)$, indicating that the two variables explained the same amount of variation in the data.

\section{Discussion}

In the present study, we investigated the contribution of selected perceptual, cognitive, and motor skills to balance control in children between the ages of 11 and 13 years. Two different balance conditions were tested. The first required unperturbed standing balance under static conditions (quiet one-leg standing), and the second involved reactions to postural self-induced disturbances evoked by rapid hip flexion-extension or abduction-adduction. The results confirmed the hypothesis that, depending on the imposed environmental constraints and nature of the balance task, static or dynamic, the selection of the appropriate balance strategy and therefore the perceptual, motor, and cognitive processes involved in maintaining equilibrium can be quite different (Assaiante \& Amblard, 1995; Haas \& Diener, 1988). Moreover, we found that 11- to 13-year-old children are capable of applying strategies that significantly resemble those used by adults for maintaining equilibrium under static or dynamic conditions.

The significant relationship of static balance with depth perception and with visuomotor coordination suggests that one-leg standing is regulated by a feedback-based mechanism in which sensory information from visual cues becomes important for the continuous adjustments; moreover, corrections of the required posture that are needed so that the COM can be kept within the narrow boundaries defined by the base of support. Other investigators (Ghez, 1991; Nashner, 1976; Robertson, Collins, Elliott, \& Starkes, 1994) have proposed a slow sensory integration process such as the one just described, which is based on a closedloop system, as the regulating mechanism for quiet stance. In addition, an increasing body of evidence suggests that information processed through vision, particularly from peripheral visual cues providing exteroceptive information about the environment, is the most reliable source of perceptual information for balance control, especially in children (Assaiante \& Amblard, 1995; Garland \& Barry, 1990; Grasso et al., 1998; Riach \& Hayes, 1990; Williams, Davids, Burwitz, \& Williams, 1994). However, the ability to interpret and use peripheral visual cues is associated with increasing accuracy and consistency of eye movements, which is acquired with age (Starkes \& Riach, 1990) and is not achievable in younger children. For that reason, children between 6 and 7 years of age rely mostly on vestibular information about head position relative to the supporting surface; that information becomes progressively more available to the equilibrium control centers as a result of the acquisition of the head stabilization in space strategy (Assaiante \& Amblard, 1995; Assaiante, Marchand, \& Amblard, 1989; Forssberg \& Nashner, 1982). The relative contribution of peripheral vision in equilibrium control increases from 8 to 9 years of age to adulthood (Assaiante et al., 1989). In adults, peripheral visual cues as well as visual motion cues have been reported to play a particularly important role in postural control (Amblard, Crémieux, Marchand, \& Carblanc, 1985). The results of the present study confirmed the important role of peripheral visual cues for postural stabilization in 11- to 13-year-old children as well. In addition to that finding, we showed that the relationship between balance and visual motion cues was significant only under conditions involving motion of the experimental dowel in the backward direction. That finding may indicate the higher contribution of vision during corrective backward body sway, when visual cues appear to be moving away from the eyes. Exteroceptive information can be available through the kinesthetic system as well, although it has been suggested that vision provides an easier anchoring for that information to the gravito-inertia reference frame (Patla, 1999). In the present study, poor correlation of kinesthesis with both static and dynamic balance 
measures was observed. That finding can be attributed to the fact that all balance exercises were performed with eyes open, although, on the other hand, kinesthesis was tested in the absence of vision. The predominance of visual over proprioceptive cues for static and dynamic equilibrium is not new (Assaiante \& Amblard, 1995; Garland \& Barry, 1990; Williams et al., 1994). We speculate that under the conditions involving vision, the afferent contributions of the kinesthetic system to static balance control are seriously limited.

In contrast to one-leg quiet stance, in which a significant reliance on visual cues was found, and in accordance with previous studies (Do, Brenière, \& Brenguier, 1982; Woollacott et al., 1986), balance performance under dynamic conditions involving a self-induced perturbation in the $\mathrm{M} / \mathrm{L}$ plane was predominantly associated with an adequately functioning reaction time process. Short reaction times are known to be associated with preparatory adjustments in a given perturbation and to reduce the disturbance of the COM during performance of postural tasks (Riach \& Hayes, 1987). According to the results of the present study, the ability to rapidly respond to a destabilization induced by rapid hip abduction-adduction in order to maintain equilibrium is greatly dependent on reaction time. That finding suggests that postural reactions to rapid hip abductions-adductions are preprogrammed in an open-loop fashion, whereas the child has the ability to apply feedforward control in his other movement and therefore to reliably coordinate posture with the performed task. Balance control in that case is temporally organized in a descending order, from head to toe (Assaiante \& Amblard, 1995). Recent experimental evidence suggests that such an anticipatory, open-loop (feedforward) mode of balance control appears at around the age of 7 years and continues through adulthood (Grasso et al., 1998; Ledebt, Bril, \& Brenière, 1998). In contrast, a feedback-based control corresponding to an ascending organization of balance control from foot to head (in posture) and hip to head (in locomotion) is more characteristic of children under the age of 6 (Assaiante \& Amblard, 1995). According to the same authors, the ascending, descending, and mixed strategies all co-exist in adults, and the selection of one or another and their relevant contributions greatly depend on the particular task conditions. For example, in difficult balance situations, the descending strategy (from head to toe) is always dominant. On the basis of our present findings, we speculate that dominance of that strategy is also true for children between the ages of 11 and 13 years, when, according to studies reported in the literature, the complexity of the CNS with respect to balance control strongly resembles that of an adult (Berger et al., 1987; Ledebt et al., 1998). We showed that balancing under static conditions was strongly associated with the ability to perceive and process visual information, an ability that is important for feedback-based control of balance (ascending strategy). On the other hand, when greater task demands were imposed on the system under dynamic balancing con- ditions, the ability to respond to the destabilizing hip abductions-adductions in order to maintain equilibrium was associated with the motor response speed, implying the possible involvement of a descending, feedforward strategy in the control of balance. The significant association of dynamic balance with discrimination reaction time suggests, however, that although postural reactions to hip abduction-adduction perturbations seem to be preprogrammed in an openloop fashion, the use of sensory information or cognitive processing at a later stage to modulate the response is not precluded. Further evidence in support of cognitive contributions to the control of both static and dynamic balance was provided by the significant relationship between the numbers of errors in the choice reaction time test and in both static and dynamic balance indices. Because the number of incorrect responses in the choice reaction time test represented the speed of information processing in the response selection stage, that relationship suggests that cognitive interventions targeted to speed up processes of a certain stage may also be involved in both static and dynamic equilibrium control (Thomas, Thomas, \& Gallagher, 1993). Those findings are in agreement with previous studies showing that postural control might rely not only on peripheral sensory information but also on higher-level systems responsible for integrating sensory information (Teasdale et al., 1992; Woollacott et al., 1992; Woollacott et al., 1986). There is no doubt, however, that the relationship between postural control and cognitive processes is rather complex, and researchers must perform further research to substantiate any findings concerning cognitive contributions to postural control.

Another interesting finding of the present study is that motor response speed, as reflected in reaction time measures, seems to be an important element in dynamic balance control only when postural disturbance occurs in the frontal $(\mathrm{M} / \mathrm{L})$ plane. In contrast, when destabilization occurred in the $\mathrm{A} / \mathrm{P}$ plane because of rapid hip flexion-extension, no association between the amplitude of the COM oscillation and reaction time was noted. There are several possible reasons for that task-specific contribution of reaction time in dynamic balance control. First of all, reactive control of balance, through a reflex-activated feedback loop, might not be the only mechanism used by the CNS to control the position of the COM in response to external perturbations (Rietdyk, Patla, Winter, Ishac, \& Little, 1999). Alternatively, it is possible that the CNS uses muscle tone to set joint stiffness to control body posture. In the sagittal plane, there is stiffness control at the ankle plantarflexors, whereas in the frontal plane, the hip abductors-adductors provide that control. On the basis of those findings, we speculate that the inherent properties of the muscles and passive tissue around the ankle could have provided an immediate resistance to the A/P perturbation, eliminating the need for voluntary active muscle activation during performance of the flexion-extension dynamic exercise. Riach and Hayes (1987), who have also shown that the maturation of postural responses in the 
two planes may follow different courses in children, provided further evidence in support of that hypothesis. That conclusion was based on experimental evidence showing that the initiation of APAs was more often in the lateral than in the sagittal plane, indicating the stronger association between reaction time and balance control in the lateral plane.

\section{Conclusion}

Balance control during performance of different posturokinetic activities in children not only is an age-dependent process associated with neural maturation but is also associated with task or environmental constraints. The results of the present study suggest that, like adults, 11- to 13-yearold children have the ability to select varying balance strategies depending on the constraints of a particular task. Balancing under static conditions was shown to be greatly associated with the ability to perceive and process visual information, an ability that is important for feedback-based control of balance. On the other hand, when greater task demands were imposed on the system under dynamic balancing conditions, the ability to respond to the destabilizing hip abductions-adductions so as to maintain equilibrium was associated with motor response speed, implying the possible involvement of a descending feedforward mechanism in the control of balance.

\section{ACKNOWLEDGMENT}

The authors thank Patricia McKinley as well as the two anonymous reviewers for their helpful comments and suggestions on an earlier draft of the present article. The present study was partially supported by a research grant from the National Scholarships Foundation.

\section{REFERENCES}

Amblard, B., Crémieux, J., Marchand, A. R., \& Carblanc, A. (1985). Lateral orientation and stabilization of human stance: Static versus dynamic visual cues. Experimental Brain Research, 61, 21-37.

Assaiante, C., \& Amblard, B. (1992). Peripheral vision and agerelated differences in dynamic balance. Human Movement Science, 11, 533-548.

Assaiante, C., \& Amblard, B. (1995). An ontogenetic model for the sensorimotor organization of balance control in humans. Human Movement Science, 14, 13-43.

Assaiante, C., Marchand, A. R., \& Amblard, B. (1989). Discrete visual samples may control locomotor equilibrium and foot positioning in man. Journal of Motor Behavior, 21, 72-91.

Berger, W., Quintern, J., \& Dietz, V. (1987). Afferent and efferent control of stance and gait: Developmental changes in children. Electroencephalography and Clinical Neurophysiology, 66, 244-252.

Caron, O., Faure, B., \& Brenière, Y. (1997). Estimating the centre of gravity of the body on the basis of the centre of pressure in standing posture. Journal of Biomechanics, 30, 1169-1171.

Do, M. C., Brenière, Y., \& Brenguier, P. (1982). A biomechanical study of balance recovery during the fall forward. Journal of Biomechanics, 15, 933-939.

Forssberg, H., \& Nashner, L. M. (1982). Ontogenetic development of postural control in man: Adaptation to altered support and visual conditions during stance. Journal of Neuroscience, 2, $545-552$.

Gallahue, D. L., \& Ozmun, J. C. (1998). Understanding motor development (2nd ed.). Dubuque, IA: Brown and Benchmark.

Garland, D. J., \& Barry, J. R. (1990). Sport expertise: The cognitive advantage. Perceptual and Motor Skills, 70, 1299-1314.

Ghez, C. (1991). Posture. In E. Kandel, T. Schwartz, \& M. Jessel (Eds.), Principles of neural science (pp. 596-607). New York: Elsevier Science.

Grasso, R., Assaiante, C., Prevost, P., \& Berthoz, A. (1998). Development of anticipatory orienting strategies during locomotor tasks in children. Neuroscience Biobehavioral Reviews, 22, 533-539.

Haas, G., \& Diener, H. C. (1988). Development of stance control in children. In B. Amblard, A. Berthoz, \& F. Clarac (Eds.), Posture and gait: Development, adaptation and modulation (pp. 49-58). Amsterdam: Elsevier.

Horak, F. B., \& Nashner, L. M. (1986). Central programming of postural movements: Adaptations to altered support surface configurations. Journal of Neurophysiology, 55, 1369-1381.

Jeka, J., Oie, K. S., \& Kiemel, T. (2000). Multisensory information for human postural control: Integrating touch and vision. Experimental Brain Research, 134, 107-125.

Kavounoudias, A., Gilhodes, J. C., Roll, R., \& Roll, J. P. (1999). From balance regulation to body orientation: Two goals for muscle proprioceptive information processing? Experimental Brain Research, 124, 80-88.

Lacquaniti, F., \& Maioli, C. (1989). The role of preparation in tuning anticipatory and reflex responses during catching. Journal of Neuroscience, 9, 134-148.

Ledebt, A., Bril, B., \& Brenière, Y. (1998). The build-up of anticipatory behaviour. An analysis of the development of gait initiation in children. Experimental Brain Research, 120, 9-17.

Lord, S. R., Clark, R. D., \& Webster, I. W. (1991). Visual acuity and contrast to sensitivity in relation to falls in an elderly population. Age and Ageing, 20, 175-181.

Massion, J. (1992). Movement, posture and equilibrium: Interaction and coordination. Progress in Neurobiology, 38, 35-56.

Massion, J., \& Woollacott, M. (1996). Posture and equilibrium. In A. Bronstein, T. Brandt, \& M. Woollacott (Eds.), Clinical disorders of balance, posture and gait (pp. 1-18). London: Oxford University Press.

Nashner, L. M. (1976). Adapting reflexes controlling the human posture. Experimental Brain Research, 26, 59-72.

Riach, C. L., \& Hayes, K. C. (1987). Maturation of postural sway in young children. Developmental Medicine and Child Neurology, 29, 650-658.

Riach, C. L., \& Hayes, K. C. (1990). Anticipatory postural control in children. Journal of Motor Behavior, 22, 250-266.

Rietdyk, S., Patla, A. E., Winter, D. A., Ishac, M. G., \& Little, C. E. (1999). Balance recovery from medio-lateral perturbations of the upper body during standing. Journal of Biomechanics, 32, $1149-1158$.

Robertson, S., Collins, J., Elliott, D., \& Starkes, J. (1994). The influence of skill and intermittent vision on dynamic balance. Journal of Motor Behavior, 26, 333-339.

Roders, M. W., Wardman, D. L., Lord, S. R., \& Fitzpatrick, R. C. (2001). Passive tactile sensory input improves stability during standing. Experimental Brain Research, 136(4), 514-522.

Schmidt, R. A. (1982). Motor control and learning: A behavioral emphasis. Champaign, IL: Human Kinetics.

Schmitz, C., Martin, N., \& Assaiante, C. (1999). Development of anticipatory postural adjustments in a bimanual load-lifting task in children. Experimental Brain Research, 126, 200-204.

Schuhfried, G. (1996). Manuals of the VIENNA Test Instrument Systems. Mödling, Austria: Schufried Eigenverlag. 
Shimba, T. (1984). An estimation of center of gravity from force platform data. Journal of Biomechanics, 17, 53-60.

Shumway-Cook, A., \& Woollacott, M. (1985). The growth of stability: Postural control from a developmental perspective. Journal of Motor Behavior, 17, 131-147.

Shumway-Cook, A., \& Woollacott, M. (2000). Attentional demands and postural control: The effect of sensory context. Journal of Gerontology A: Biological Science Medical Science, 55(1), M10-6.

Slijper, H., \& Latash, M. (2000). The effects of instability and additional hand support on anticipatory postural adjustments in leg, trunk and arm muscles during standing. Experimental Brain Research, 135, 81-93.

Starkes, J., \& Riach, C. (1990). The role of vision in the postural control of children. Clinical Kinesiology, 44, 72-77.

Sundermier, L., \& Woollacott, M. H. (1998). The influence of vision on the automatic postural muscle responses of newly standing and newly walking infants. Experimental Brain Research, 120(4), 537-540.

Sundermier, L., Woollacott, M., Roncesvalles, N., \& Jensen, J. (2000). The development of balance control in children: Comparisons of EMG and kinetic variables and chronological and developmental groupings. Experimental Brain Research, 136(3), 340-350.

Sveistrup, H., \& Woollacott, M. H. (1996). Longitudinal development of the automatic postural response in infants. Journal of Motor Behavior, 28, 58-70.

Teasdale, N., Lajoie, Y., Bard, C., Fleury, M., \& Courtemanche, R. (1992). Cognitive process involved for maintaining postural stability while standing and walking. G. E. Stelmach \& V. Homberg (Eds.), Sensorimotor impairment in the elderly (pp. 157-168). Amsterdam: Kluwer Academic.

Thomas, J. R., Thomas, K. T., \& Gallagher, J. D. (1993). Developmental considerations in skill acquisition. In $\mathrm{R}$.
Singer, M. Murphey, \& K. Tennant (Eds.), Handbook of research on sport psychology (pp. 73-105). New York: Macmillan.

Werder, J. K., \& Bruininks, R. H. (1988). Body skills: A motor development curriculum for children. New York: American Guidance Service.

Williams, M., Davids, K., Burwitz, L., \& Williams, J. (1994). Visual search strategies in experienced and inexperienced soccer players. Research Quarterly for Exercise and Sport, 65, 127-135.

Winter, D. A., Patla, A. E., Prince, F., Ishac, M., \& Gielo-Perczak, K. (1998). Stiffness control of balance in quiet standing. Journal of Neurophysiology, 80, 1211-1221.

Woollacott, M. H. (1988). Posture and gait from newborn to elderly. In B. Amblard, A. Berthoz, \& F. Clarac (Eds.), Posture and gait: Developmental adaptation and modulation ( $p p$ 423-432). Amsterdam: Elsevier Science.

Woollacott, M. H., Moore, S., \& Hu, M. H. (1992). Improvements in balance in the elderly through training in sensory organization abilities. In G. E. Stelmach \& V. Homberg (Eds.), Sensorimotor impairment in the elderly (pp. 377-392). Amsterdam: Kluwer.

Woollacott, M. H., Shumway-Cook, A., \& Nashner, L. M. (1986). Aging and posture control: Changes in sensory organization and muscular coordination. International Journal of Aging and Human Development, 23, 97-114.

Zatsiorsky, V. M., \& King, D. L. (1998). An algorithm for determining gravity line location from posturographic recordings. Journal of Biomechanics, 31, 161-164.

Submitted December 23, 1999

Revised October 11, 2000

Second revision March 1, 2001 